\title{
TVDI Obtido de Imagens OLI/TIRS e MODIS
}

\author{
Lucimara Wolfarth Schirmbeck $^{1}$ (D), Denise Cybis Fontana ${ }^{2}$ (D), Juliano Schirmbeck ${ }^{1}$ (D), \\ Carolina Bremm ${ }^{3}$ \\ ${ }^{1}$ Programa de Pós-Graduação em Sensoriamento Remoto Centro Estadual de Pesquisas em \\ Sensoriamento Remoto e Meteorologia, Universidade Federal do Rio Grande do Sul, Porto \\ Alegre, RS, Brasil. \\ ${ }^{2}$ Departamento de Plantas Forrageiras e Agrometeorologia, Faculdade de Agronomia, \\ Universidade Federal do Rio Grande do Sul, Porto Alegre, RS, Brasil. \\ ${ }^{3}$ Departamento de Diagnóstico e Pesquisa Agropecuária, Secretaria da Agricultura, Pecuária e \\ Irrigação, Porto Alegre, RS, Brasil.
}

Recebido em: 25 de Julho de 2018 - Aceito em: 18 de Dezembro de 2018

\begin{abstract}
Resumo
O objetivo do estudo foi analisar a variabilidade no TVDI (Temperature-Vegetation Dryness Index) obtido de sensores orbitais com resoluções distintas, em região agrícola no sul do Brasil. Utilizou-se três imagens OLI/TIRS (Operational Land Imager/Thermal Infrared Sensor) do satélite Landsat 8, e 12 imagens MODIS (Moderate Resolution Imaging Spectroradiometer) do satélite Terra. Dados coletados em campo serviram como base para classificação de imagem OLI/TIRS e mapeamento de áreas de arroz, soja, campos naturais, mata ciliar e solo exposto. O TVDI foi obtido por duas parametrizações em períodos distintos, utilizando as dispersões entre Temperatura de Superfície $\left(T_{S}\right)$ e NDVI (Normalized Difference Vegetation Index). O TVDI obtido para ambos sensores apresentou padrão similar possibilitando diferenciar os alvos. Na média de todas as datas e classes, o TVDI obtido das imagens MODIS foi superior em 0,128 unidades ao TVDI obtido com o OLI/TIRS. Quando utilizado OLI/TIRS há um melhor detalhamento espacial das condições hídricas, mas com menor repetição ao longo da safra; já utilizando o TVDI-MODIS é possível monitorar as condições hídricas em escala regional, com menor detalhamento espacial, mas com maior repetitividade no tempo. $\mathrm{O}$ TVDI estimado pelos sensores OLI/TIRS e MODIS, pode ser utilizado de forma conjunta, trazendo informações complementares.
\end{abstract}

Palavras-chave: temperatura de superfície, NDVI, agricultura, déficit hídrico.

\section{TVDI from OLI/TIRS and MODIS images}

\begin{abstract}
The aim of the study was to analyze the variability in the TVDI (Temperature-Vegetation Dryness Index) obtained from orbital sensors with distinct resolution in an agricultural region in southern Brazil. Three images of the Landsat 8 satellite OLI/TIRS (Operational Land Imager/Thermal Infrared Sensor) and 12 of Terra's MODIS (Moderate Resolution Imaging Spectroradiometer), were used. Data collected in the field served as a basis for OLI/TIRS image classification and mapping of rice, soybean, grasslands, gallery forest and bare soil areas. The TVDI obtained by two parameterizations at different periods was evaluated, using the dispersions between Surface Temperature $\left(T_{S}\right)$ and NDVI (Normalized Difference Vegetation Index). The TVDI obtained for both sensors presented a similar pattern allowing differentiation of the targets. The average of all dates and classes, TVDI obtained from MODIS was 0.128 units higher than the obtained with the OLI/TIRS. When used OLI/TIRS there is a better detail in the representation of the moisture, but with less repetition throughout the crop cycle. Using TVDI-MODIS, it is possible to monitor moisture conditions on a regional scale, with less spatial detail, but in a continuous way over time. The TVDI estimated by the OLI/TIRS and MODIS can be used together, providing complementary information.
\end{abstract}

Keywords: Surface temperature, NDVI, agriculture, water deficit.

Autor de correspondência: Lucimara Wolfarth Schirmbeck, lucimaraws@gmail.com. 


\section{Introdução}

O déficit hídrico é um fenômeno que influencia tanto questões econômicas quanto ambientais, estando no Rio Grande do Sul (RS) associado a grandes perdas na produção agrícola (EMATER/RS, 2018). Os déficits no RS ocorrem especialmente no período de primavera-verão, visto que grande parte da agricultura é conduzida sem irrigação. Neste período de alta demanda evaporativa da atmosfera, seguidamente a oferta de precipitação pluvial não é suficiente para atender às necessidades hídricas das plantas. Agregam-se a isto, à alta variabilidade da precipitação pluvial entre uma safra e outra, a qual, em parte, está associada a eventos de grande escala como El Niño Oscilação Sul (ENOS). Geralmente no Rio Grande do Sul, anos de baixa e alta precipitação pluvial na primaveraverão estão associados a eventos de La Niña e a de El Niño, respectivamente (Berlato e Cordeiro, 2017).

Apesar da relevância do tema, é escassa a disponibilidade de indicadores que permitam obter informações sobre a umidade da superfície de grandes áreas de produção, com adequado nível de detalhamento espacial e com a frequência de cobertura temporal compatível com o ciclo de crescimento e desenvolvimento das plantas. As imagens de satélite podem suprir parte da lacuna na obtenção de indicadores para o gerenciamento racional do uso da água. Um exemplo, é a estimativa da umidade da superfície (Chen et al. 2015; Sayago et al. 2017), a qual pode ser obtida de forma indireta através de um índice, o TVDI (Temperature-Vegetation Dryness Index) (Sandholt et al. 2002).

Neste índice, são usados de forma conjugada, dados de temperatura de superfície $\left(T_{S}\right)$ e de índice de vegetação (IV), ambos em um espaço bidimensional, fornecendo dados que permitem avaliar a variabilidade da umidade do solo, e com repetições frequentes ao longo do tempo. $\mathrm{O}$ TVDI tem sido aplicado com sucesso em várias regiões e para diferentes tipos de imagens de satélite e tempo de aquisição (Sandholt et al. 2002; Wang et al. 2004; Mallick et al. 2009; Holzman et al. 2014; Chen et al. 2015; Uniyal et al. 2017; Sayago et al. 2017; Schirmbeck et al. 2018).

No TVDI, a $T_{S}$ indica o estado hídrico das plantas, visto que, se a planta não consegue atender a demanda evaporativa da atmosfera a $T_{S}$ é maior do que a temperatura do ar. O IV indica a condição e variabilidade da biomassa verde. A inclinação negativa da reta da dispersão que ocorre no espaço $T_{S} / \mathrm{IV}$ está relacionada à taxa de evapotranspiração da superfície, sendo usada, portanto, para parametrizar o modelo que permite avaliar as condições da umidade do solo (Chen et al. 2015). Salienta-se, entretanto, que o TVDI possui importante dependência desta parametrização (Schirmbeck et al. 2018) e a correta compreensão do significado deste índice depende da forma como o mesmo é obtido. O TVDI é, portanto, um valor relativo às condições de umidade da área em um determi- nado período. Alterando qualquer destes dois fatores (área e período), altera o valor do índice, o que para estudos multitemporais introduz certa complexidade, que por vezes passa desapercebida aos usuários.

O uso de diferentes sensores para a obtenção do TVDI, pode gerar incertezas que decorrem de diferenças nas resoluções espaciais, espectrais e temporais dos mesmos, as quais precisam ser avaliadas e dimensionadas. Considerando os sensores atualmente disponíveis para geração de informações úteis para o setor agrícola, possivelmente sejam o OLI/TIRS (Operational Land Imager) Thermal Infrared Sensor) e o MODIS (Moderate Resolution Imaging Spectroradiometer), os mais intensamente utilizados. Isto porque existe certa complementariedade, onde o primeiro permite maior detalhamento espacial e o segundo maior detalhamento temporal, ambos atributos bastante relevantes. Também relevante é mencionar que ambos possuem bandas posicionadas em faixas do espectro que permitem a determinação do TVDI, o que justifica a presente investigação.

Tendo em vista a dificuldade de dispor de medições da umidade da superfície com adequada representação espacial, o objetivo do presente estudo foi analisar os valores de TVDI obtido pelos sensores OLI/TIRS e MODIS com resoluções espacial, espectral e temporal distintas, assim como, analisar a consistência na sua distribuição espacial em região de produção agrícola localizada no RS.

\section{Material e Métodos}

A área de estudo está localizada no Estado do Rio Grande do Sul, Brasil, na unidade geomorfológica da Depressão Central, em altitudes que variam entre $100 \mathrm{e}$ $300 \mathrm{~m}$ e com predominância de atividades agrícolas e campos naturais do Bioma Pampa (Guasselli et al. 2006). O clima, segundo Alvares et al. (2013), com base na classificação climática de Köppen, é o tipo climático Cfa (clima temperado úmido com verão quente).

Com um total de cerca de 46 mil hectares, a área de estudo se localiza nos municípios de Jaguari, Mata, São Vicente do Sul e São Pedro do Sul (Fig. 1), num total de 270.180 pixels mapeados na imagem OLI/TIRS nas seguintes classes: áreas de arroz (28.996 pixels), campos (186.594 pixels), mata ciliar (10.153 pixels), soja (36.022 pixels) e solo exposto (8.415 pixels). Os dados de localização destas classes foram coletados in situ em março de 2015 em um levantamento de campo utilizando receptor GPS de navegação. Estes pontos serviram como pontos de controle para a classificação de toda a imagem.

Foi calculado o balanço hídrico meteorológico utilizando a metodologia proposta por Thornthwaite-Mather (1955) para o período das safras das culturas de primavera-verão de novembro de 2014 a abril de 2015. Para tanto foram utilizados os dados meteorológicos da estação 

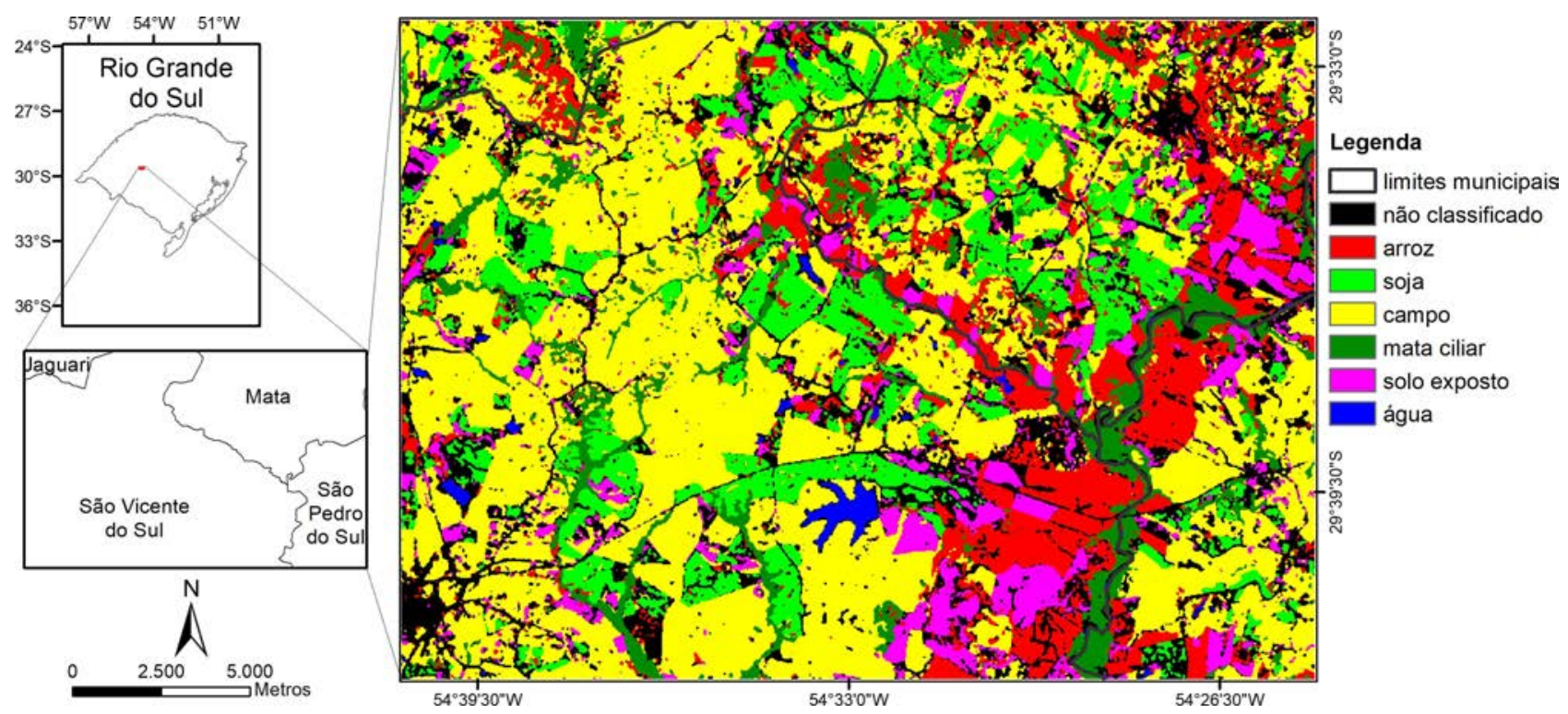

Figura 1 - Delimitação da área de estudo no estado do Rio Grande do Sul com localização dos municípios. Classificação de Imagem OLI/TIRS - Landsat 8 de 07 de fevereiro de 2015. Órbita/Ponto: 223/81.

de Santa Maria $\left(-29,7^{\circ} \mathrm{S}\right.$ e $-53,7^{\circ} \mathrm{W} ; 95 \mathrm{~m}$ de altitude), a mais próxima da área de estudo, onde se obteve dados de déficit hídrico. Para compatibilizar com a escala temporal das imagens MODIS, o déficit hídrico foi acumulado no período de 16 dias, enquanto para OLI/TIRS foram utilizados os dados diários (dia da passagem).

O TVDI, proposto por Sandholt et al. (2002), foi obtido através da relação estabelecida no espaço bidimensional entre a $T_{S}$ e o IV Eq. (1). O índice pode variar de zero a 1 , sendo que quanto mais próximo de 1 maior a restrição hídrica.

$$
T V D I=\frac{\left(T_{S}-T_{\text {Smin }}\right)}{\left(a+b I V-T_{\text {Smin }}\right)}
$$

sendo: $T_{S}$ a temperatura radiativa do pixel $(\mathrm{K}) ; T_{S \sin }$ a temperatura mínima de superfície $(\mathrm{K})$ correspondente ao limite úmido na dispersão do triângulo evaporativo; $I V \mathrm{o}$ índice de vegetação; " $a$ " e " $b$ " os coeficientes linear e angular da reta que representam o limite seco obtidos a partir do gráfico de dispersão entre o $I V$ e a $T_{S}$.

As diferenças entre escalas de aquisição tanto espacial e espectral como temporal foram analisadas a partir do uso de imagens dos sensores OLI/TIRS do satélite Landsat 8 e MODIS do satélite Terra.

\subsection{Sensor OLI/TIRS - Landsat 8}

As imagens OLI/TIRS do satélite Landsat 8 foram obtidas através da base de dados do USGS - ESPA (United States Geological Survey - Center Science Processing Architecture), numa resolução de $30 \mathrm{~m}$ nas bandas do visível e do infravermelho próximo e $100 \mathrm{~m}$ nas bandas termais, da órbita ponto 223/81. Em função de ruídos, nuvens e vapor d'água, foi possível utilizar apenas três imagens ao longo da safra de primavera-verão, abrangendo o período de implantação das culturas anuais ( 5 dez 2014), início do desenvolvimento vegetativo (6 jan 2015) e maior presença de biomassa verde ( 7 fev 2015) dos cultivos de soja e arroz no período.

Para o cálculo do TVDI-OLI/TIRS, dada a maior resolução espacial quando comparado ao sensor MODIS, a seleção dos pixels quentes e frios para cálculo do índice se restringiu à área de estudo.

A $T_{S}$ foi estimada a partir das bandas 10 e $11(100 \mathrm{~m})$ usando o algoritmo split-window (Eq. (2)) proposto por Jiménez-Muñoz et al. (2014) e testado no Rio Grande do Sul por (Schirmbeck et al. 2017). Este modelo proporciona melhor desempenho no processo de correção atmosférica, visto que as diferenças na constituição da atmosfera estão dentre as principais responsáveis pelas diferenças de temperatura obtidas com as 2 bandas termais usadas no algoritmo (Niclòs et al. 2007).

$$
\begin{gathered}
T_{S}=T_{10}+1,378\left(T_{10}-T_{11}\right)+0,183\left(\mathrm{~T}_{10}-\mathrm{T}_{11}\right)^{2}+ \\
-0,268+(54,3-2,238)(1-\varepsilon)+(-129,2+16,4 \omega) \Delta \varepsilon
\end{gathered}
$$

sendo $T_{10}$ e $T_{11}$ as temperaturas (K) nas bandas 10 e 11 do termal; $w$ o conteúdo de vapor de água na atmosfera $\left(\mathrm{g} \mathrm{cm}^{-2}\right)$; $\varepsilon$ a emissividade média das bandas do termal $\left(\varepsilon=0,5\left(\varepsilon_{10}\right.\right.$ $\left.+\varepsilon_{11}\right)$ ); $\Delta \varepsilon$ a diferença da emissividade nas bandas do ter$\operatorname{mal}\left(\Delta \varepsilon=\varepsilon_{10}+\varepsilon_{11}\right)$.

O índice de vegetação utilizado no cálculo do TVDIOLI/TIRS foi o NDVI, proposto por Rouse et al. (1973) obtido na base de dados do USGS - ESPA. 


\subsection{Sensor MODIS - Terra}

Para o TVDI-MODIS foram utilizadas 12 imagens em forma de produto disponível na base de dados LP DAAC (Land Processes database - Distributed Active Archive Center), com resolução espacial de $1 \mathrm{~km}$. O índice de vegetação NDVI foi obtido do produto MOD13A2, na forma de composição de máximo valor em 16 dias. O período de estudo abrangeu a safra das culturas de soja e arroz cultivadas no período de primavera-verão, de novembro de 2014 a maio de 2015.

Para a $T_{S}$ foi utilizado o produto MOD11A2, o qual é disponibilizado para períodos compostos de 8 dias. Quando comparado com o NDVI, que é disponibilizado em 16 dias, a composição da $T_{S}$ corresponde aos primeiros 8 dias (MOD13A2). Este produto de $T_{S}$ é obtido a partir de imagens nos períodos do dia e da noite, com ausência de nuvens, e geradas pelo algoritmo split-window. Foi utilizado o produto de $T_{S}$ visto a dificuldade de encontrar imagens de uma única data sem ruídos.

No caso das imagens MODIS, em função da moderada resolução espacial quando comparado ao OLI/TIRS, se adotou uma área mais ampla, abrangendo todo o Rio Grande do Sul, como área útil à seleção dos pixels quentes e frios para a parametrização e cálculo do índice.

\subsection{Parametrizações índice TVDI}

Para a parametrização foram construídos gráficos de dispersão entre a $T_{S}$ e NDVI para ambos sensores, OLI/ TIRS e MODIS (triângulos evaporativos característicos). No caso do TVDI-MODIS, foram utilizados dois períodos na parametrização:

i. dezembro/janeiro/fevereiro - para compreensão das diferenças entre os sensores;

ii. novembro a maio - para compreensão das mudanças de umidade ao longo da safra.

Utilizando a parametrização i) foi analisado o padrão de variabilidade espacial na área de estudo entre o TVDIOLI/TIRS e TVDI-MODIS. Também, as classes temáticas de soja, campos naturais, arroz, solo exposto e mata ciliar com áreas superiores a 100 ha foram vetorizadas sobre as imagens de TVDI-OLI/TIRS e TVDI-MODIS e extraídos os valores médios do índice. Foram, por fim, testadas as diferenças entre as médias para verificar a existência de diferenças significativas entre os sensores, classes e datas das imagens. Para o teste foi considerando um modelo para experimento com parcelas subsubdivididas, sendo o sensor a parcela principal, a classe a subparcela e a data de avaliação a subsubparcela. Quando detectadas diferenças entre os fatores em nível de $5 \%$ de significância $(\mathrm{p}<0,05)$, as médias foram comparadas pelo teste Tukey. Foi utilizado o programa estatístico JMP (v.13).

Já a parametrização ii) foi utilizada no sensor MODIS para obtenção dos perfis temporais médios do índice ao longo de toda a safra para as classes de soja, arroz e campos.

\section{Resultados e Discussão}

\subsection{Comparação entre período de parametrizações}

A Fig. 2 apresenta os triângulos evaporativos obtidos nas parametrizações para os dois sensores e períodos utilizados. Nestes, a temperatura mínima de superfície $\left(T_{S \sin }\right)$ define o limite úmido e quanto mais o pixel estiver próximo a este limite, mais úmida está a superfície (Carlson et al. 1994; Sandholt et al. 2002, Zhang et al. 2014). O parâmetro ' $b$ ' representa a inclinação negativa da reta obtida da dispersão de $T_{S}$ /NDVI, a qual define o limite seco e está associado à resistência dos estômatos da folha e da evapotranspiração (Nemani e Running 1989, Chen et al. 2015); quanto mais próximo deste limite o pixel estiver, mais seca é a condição da superfície (Sandholt et al. 2002; Mallick et al. 2009, Chen et al. 2015). Verifica-se que existe similaridade para as dispersões no triângulo evaporativo quando considerado o mesmo período da parametrização (dezembro-janeiro-fevereiro) para OLI/ TIRS e MODIS. A diferença mais evidente entre sensores foi que o triângulo evaporativo OLI/TIRS se localizou em uma escala de $T_{S}$ com valores um pouco superiores aos observados para o MODIS.

Os valores médios da $T_{\operatorname{Smin}}$ (Figs. 2a e 2b) foram, respectivamente $300,4 \mathrm{~K}$ e $297,4 \mathrm{~K}$, para OLI/TIRS e MODIS. Para o limite seco, a inclinação negativa foi maior para o MODIS, de -21,4, o que indica uma maior variabilidade nas condições de umidade, quando comparado ao OLI/TIRS, que foi de -20,9. $T_{\operatorname{Smin}}$ menores e maior inclinação da reta observados nas imagens MODIS possivelmente decorreram da maior área utilizada na parametrização do TVDI, visto que neste caso foi usado todo o território do RS. Dada a resolução espacial do MODIS, a área de estudo é muito restrita para gerar a variabilidade necessária a parametrização do índice, além do que o maior interesse na utilização de imagens MODIS é implementar análises com abrangência regional. Por outro lado, as temperaturas observadas no limite seco superiores no OLI/TIRS em relação ao MODIS possivelmente sejam decorrência de uma menor mistura espectral dentro do pixel. Devido as imagens serem do período de verão, as diferenças entre sensores foram mais evidentes sobre o limite seco ( $T_{S}$ máximas) do que o úmido ( $T_{S}$ mínimas).

Foi, entretanto, na comparação entre períodos de parametrização feitas para MODIS, dezembro-janeiro-fevereiro (Fig. 2b) e novembro a maio (Fig. 2c), que se observou maior diferença no formato dos triângulos evaporativos. Observa-se uma maior amplitude de variação nas $T_{S}$, dada especialmente pela menor $T_{\text {Smim }}(291,9 \mathrm{~K})$, que é coerente com o período mais prolongado utilizado nesta parametrização do MODIS. Como o TVDI é um 
a) OLI/TIRS (dez, jan e fev)

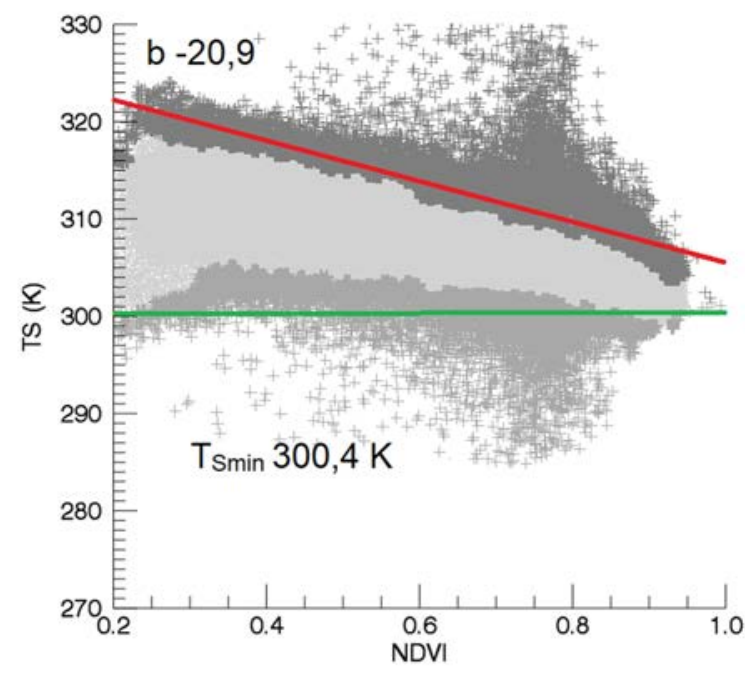

b)

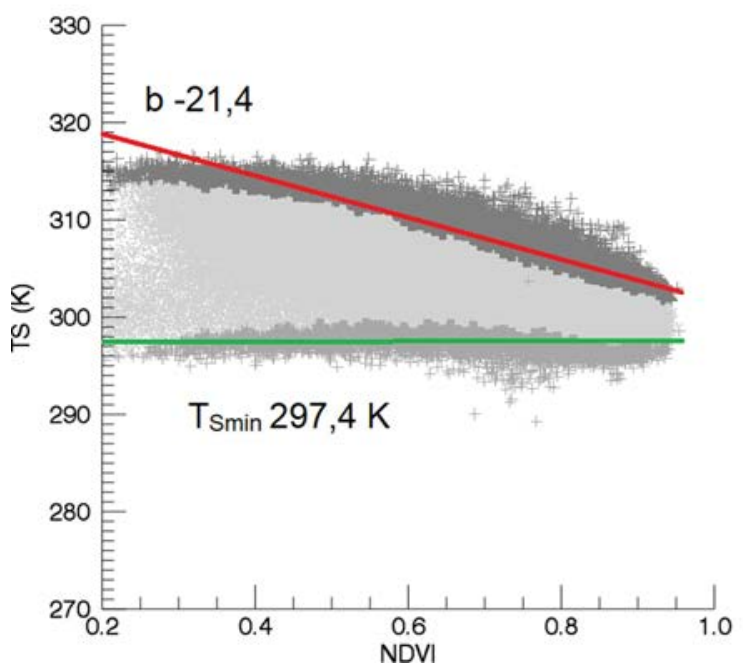

c)

MODIS (nov a maio)

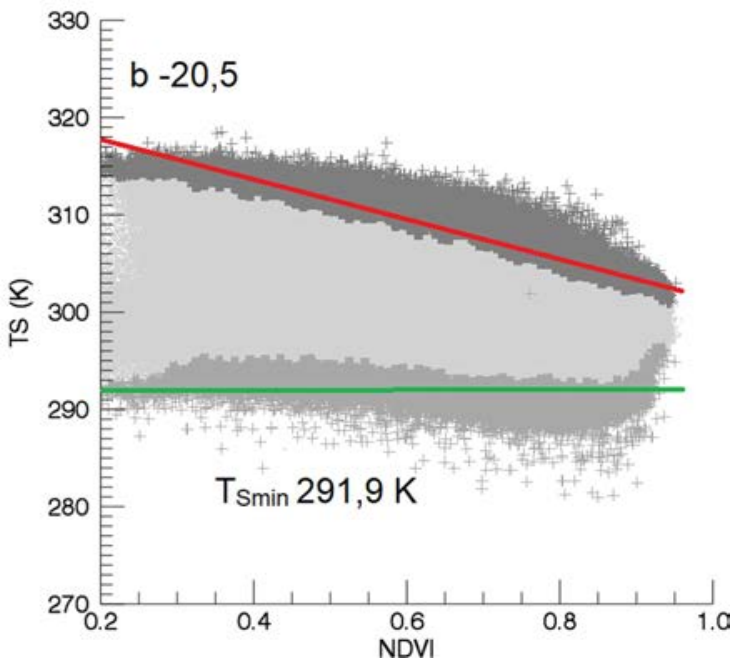

Figura 2 - Dispersão bidimensional entre a temperatura de superfície $\left(T_{S}\right)$ e o índice de vegetação (NDVI) para OLI/TIRS (a) e MODIS (b) no período de dezembro/janeiro/fevereiro, e MODIS novembro a maio (c). $T_{S \operatorname{Smin}}$ em verde é o limite úmido; inclinação negativa 'b' em vermelho é o limite seco.

índice cujos valores são relativos aos limites secos e úmidos definidos na parametrização, ressalta-se a importância da definição do período a ser utilizado, o que já foi avaliado em estudos anteriores (Schirmbeck et al. 2018).

Neste trabalho, quando as comparações foram entre sensores, utilizou-se sempre o período coincidente na parametrização (dezembro, janeiro, fevereiro). Quando o objetivo foi analisar a dinâmica temporal, foi então utilizada a parametrização abrangendo toda a safra. Na parametrização de novembro-maio estão representadas as condições de hídricas ocorridas em toda a safra (12 imagens) tornando possível, além da caracterização da condição em cada data, também a comparação entre datas. Nesta análise se dá detalhe aos extremos que ocorreram no período, possibilitando um melhor entendimento da disponibilidade hídrica ao longo do ciclo. Como comentado anteriormente, o TVDI é um índice relativo aos limites seco e úmido obtidos na parametrização. Portanto, sempre ocorrerão diferenças quando a parametrização é feita em períodos distintos. Apesar disso, o coeficiente de correlação entre o TVDI obtido nas parametrizações MODIS dezembro, janeiro e fevereiro e MODIS novembro a maio foi de 0,86 , evidenciando certa similaridade.

\subsection{Parametrização dezembro-janeiro-fevereiro - OLI/ TIRS e MODIS}

A variabilidade do TVDI na área de estudo pode ser observada na Fig. 3 para as imagens de dezembro, janeiro e fevereiro OLI/TIRS e MODIS. Apesar das diferenças entre os sensores, verifica-se que o TVDI-MODIS sempre foi superior ao TVDI-OLI/TIRS. É possível observar tam- 
OLI/TIRS

05 dez 2014

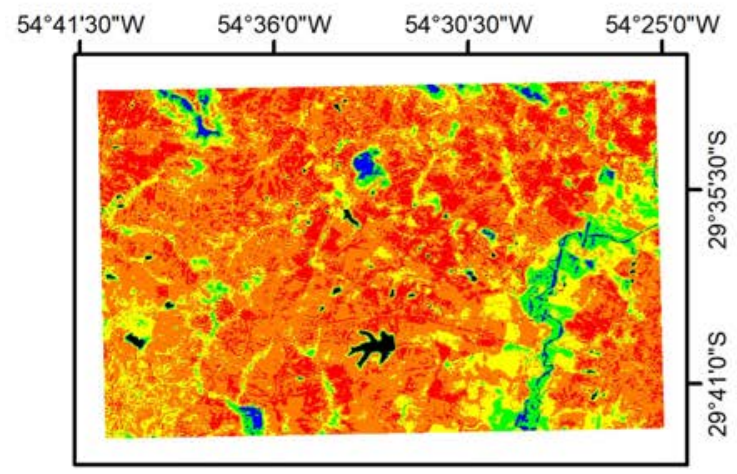

06 jan 2015

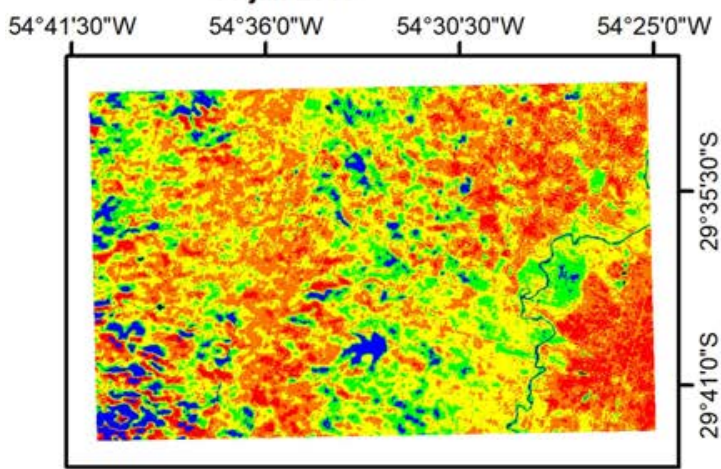

07 fev 2015
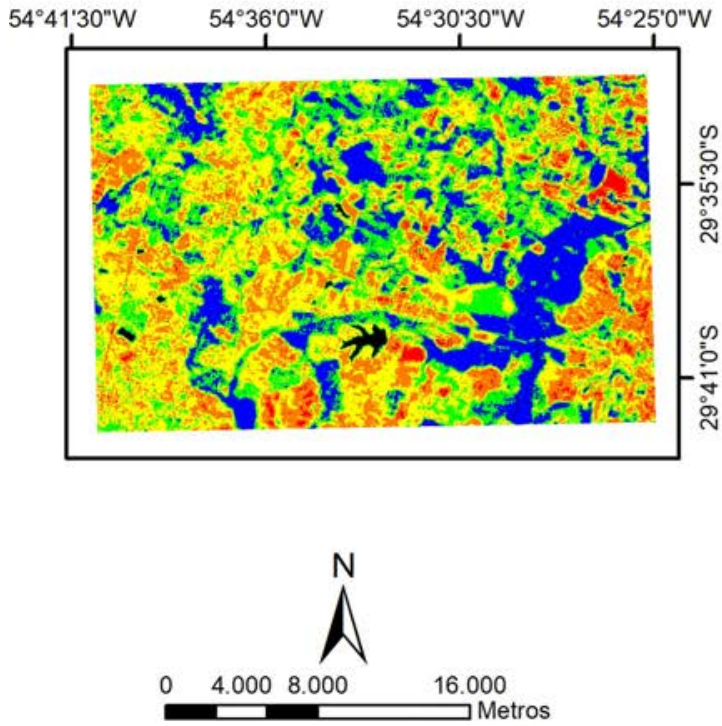

MODIS

03 - 18 dez 2014

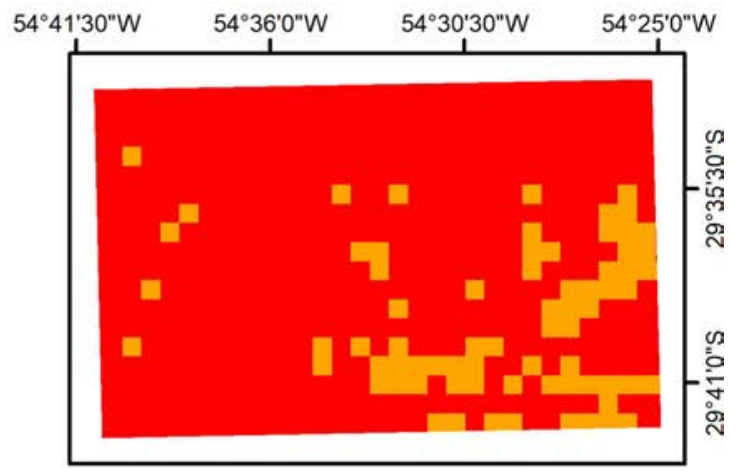

01 - 16 jan 2015

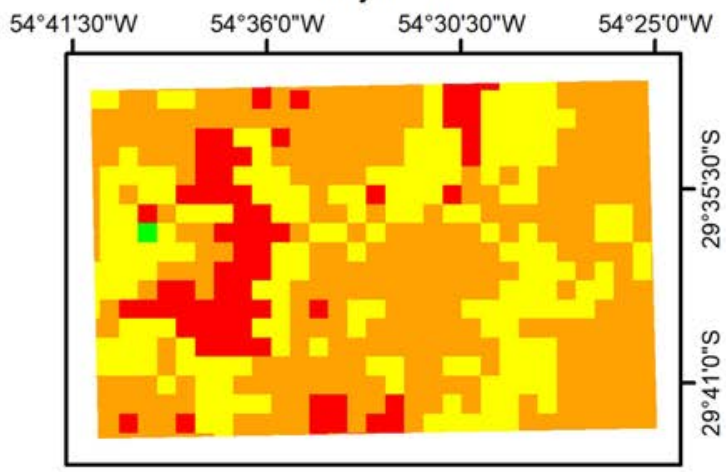

02 - 17 fev 2015

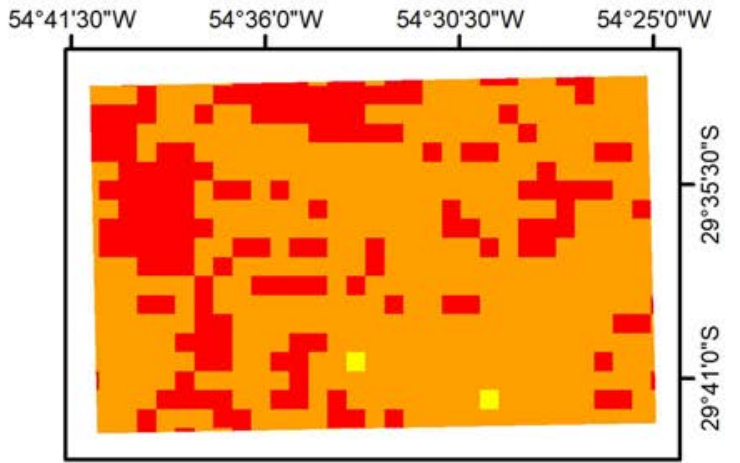

TVDI

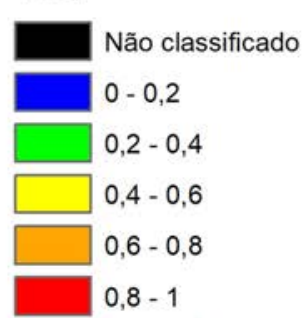

Figura 3 - Mapas da distribuição espacial do TVDI obtido a partir de dados OLI/TIRS e MODIS nos meses de dezembro de 2014, janeiro e fevereiro de 2015 na área de estudo.

bém um padrão espacial até certo ponto similar entre as imagens, coincidindo em cada data as regiões com maior e menor índice na região de estudo. Em geral, os valores mais baixos do TVDI (azul-verde) ocorrem nas áreas próximas às matas ciliares e áreas ocupadas pelas lavouras de arroz (Fig. 1), enquanto que nas áreas de campos natu- 
rais e de soja, os valores foram mais altos, indicando restrição de umidade (laranja-vermelho).

A maior resolução espacial na imagem TVDI-OLI/ TIRS determinou uma menor mistura espectral de alvos dentro do pixel e maior diferenciação entre pixels adjacentes nas imagens. Também, variações no índice possivelmente estiveram associadas às diferenças de resolução temporal, já que as imagens TVDI-OLI/TIRS são obtidas em um único dia, enquanto que as imagens TVDI-MODIS são obtidas a partir de produtos compostos de 16 dias, atenuando as diferenças.

Os valores observados para o índice TVDI mostraram coerência com a dinâmica da cobertura vegetal, assim como da condição hídrica da região. Analisando o déficit hídrico ocorrido na estação meteorológica mais próxima (Fig. 4), verifica-se que em dezembro, no período de 3 a 18/12 (período de composição dos produtos MODIS), o déficit acumulado foi de $15 \mathrm{~mm}$, intermediário quando comparado as demais datas das imagens analisadas. Apesar disso, nesta data foi quando se observou os maiores TVDI nos dois sensores. Essa aparente incoerência decorre da dinâmica da cobertura vegetal na área em estudo. Este é o período de implantação das culturas de primavera-verão, quando a maior presença de solo exposto (menor biomassa) coincide com o período de maior incidência de radiação solar (solstício de verão), determinando os altos valores da $T_{S}$, o que justifica os valores mais elevados de TVDI. Também, é importante considerar que o período anterior a este foi de acentuado déficit hídrico, o que pode ter comprometido o crescimento de biomassa.

Em contrapartida, janeiro foi o período que apresentou os menores déficits ( $7 \mathrm{~mm}$ no período de 1 a 16/01) entre as datas analisadas e, coerentemente, foram observa- das reduções nos valores de TVDI mais claramente observado nas imagens MODIS.

$\mathrm{O}$ déficit hídrico em fevereiro teve acréscimo (Fig. 4), num período caracterizado por maior presença de biomassa verde para as culturas de primavera-verão, com alta densidade de biomassa verde. Este foi o período em que se observou a maior diferença entre os dois sensores, $o$ que pode ser em parte atribuído à diferença de resolução temporal das imagens. A imagem composta de 16 dias MODIS (de 2 a 17/02) retratou o déficit acumulado no período que foi de $35 \mathrm{~mm}$. Já a imagem OLI/TIRS foi adquirida no dia 7/02 ainda no início do período de déficit, logo após ao déficit zero do final de janeiro, estando, portanto, retratando condições hídricas mais favoráveis do que as representadas nas imagens TVDI-MODIS.

Também usando a parametrização no período dezembro-janeiro-fevereiro foram comparados os valores do TVDI OLI/TIRS e MODIS separadamente para as classes arroz, soja, campos naturais, mata ciliar e solo exposto nas três datas (Fig. 5). Coerente com o observado na Fig. 3, observa-se que a maior parte dos pixels das imagens MODIS em todas as classes apresentaram valores de TVDI maiores associados à imagem de dezembro, menores valores em janeiro e valores intermediários em fevereiro. Já para o sensor OLI/TIRS ocorreu uma maior dispersão dos valores observados do índice para todas as classes e datas, o que está associado ao número muito elevado de pixels utilizados na análise, e à menor mistura espectral em função do tamanho do pixel. Soma-se a isto as possíveis diferenças decorrentes da variabilidade dentro das classes, associadas a datas de semeadura, práticas de manejo, posição no terreno, entre outros. Evidencia-se mais uma vez que com o sensor OLI/TIRS os menores

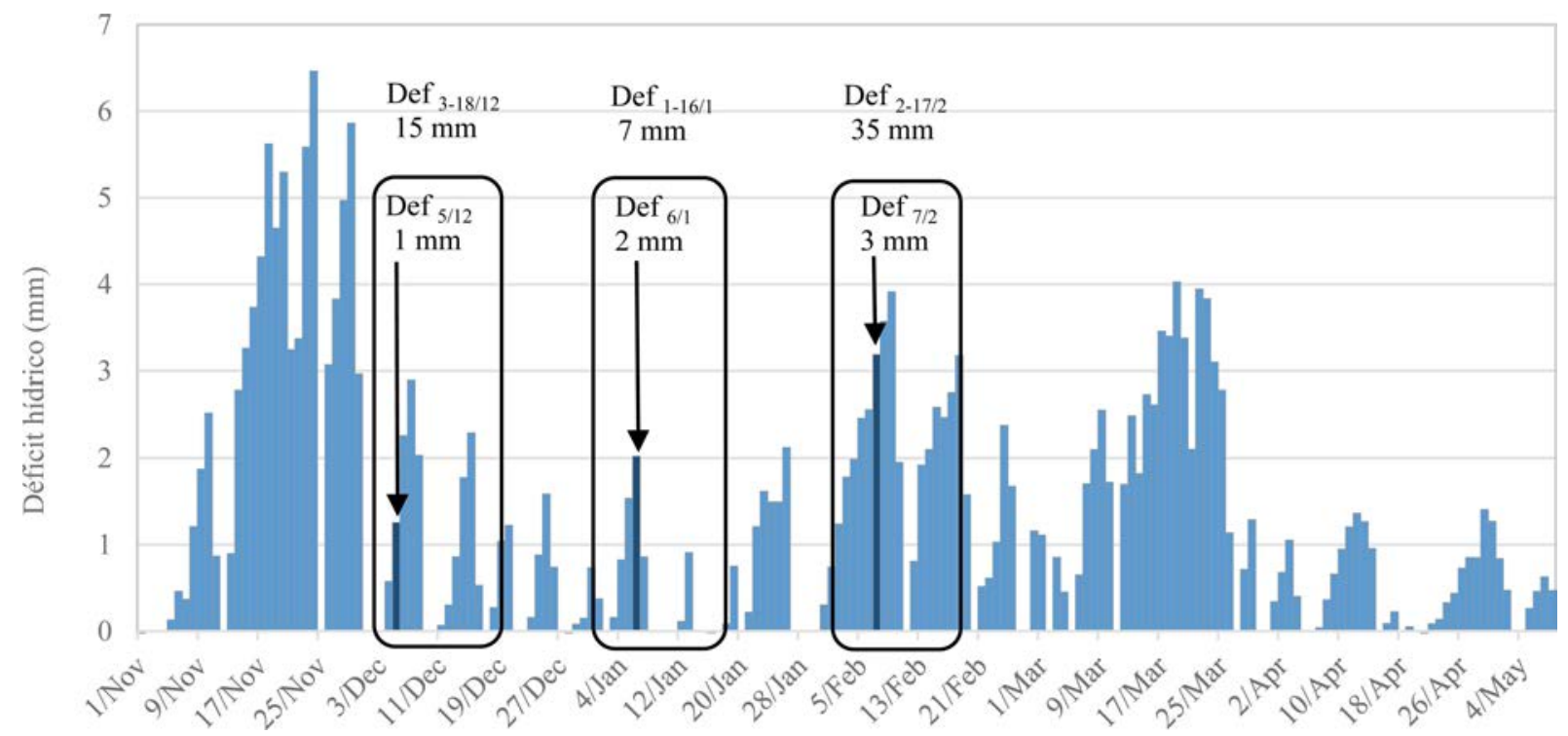

Figura 4 - Dados de déficit hídrico diário para Santa Maria no período de novembro a maio, safra 2014-2015. Os retângulos indicam o período de 16 dias utilizados na composição MODIS e a seta indica o dia da passagem OLI/TIRS, com os respectivos déficits. 


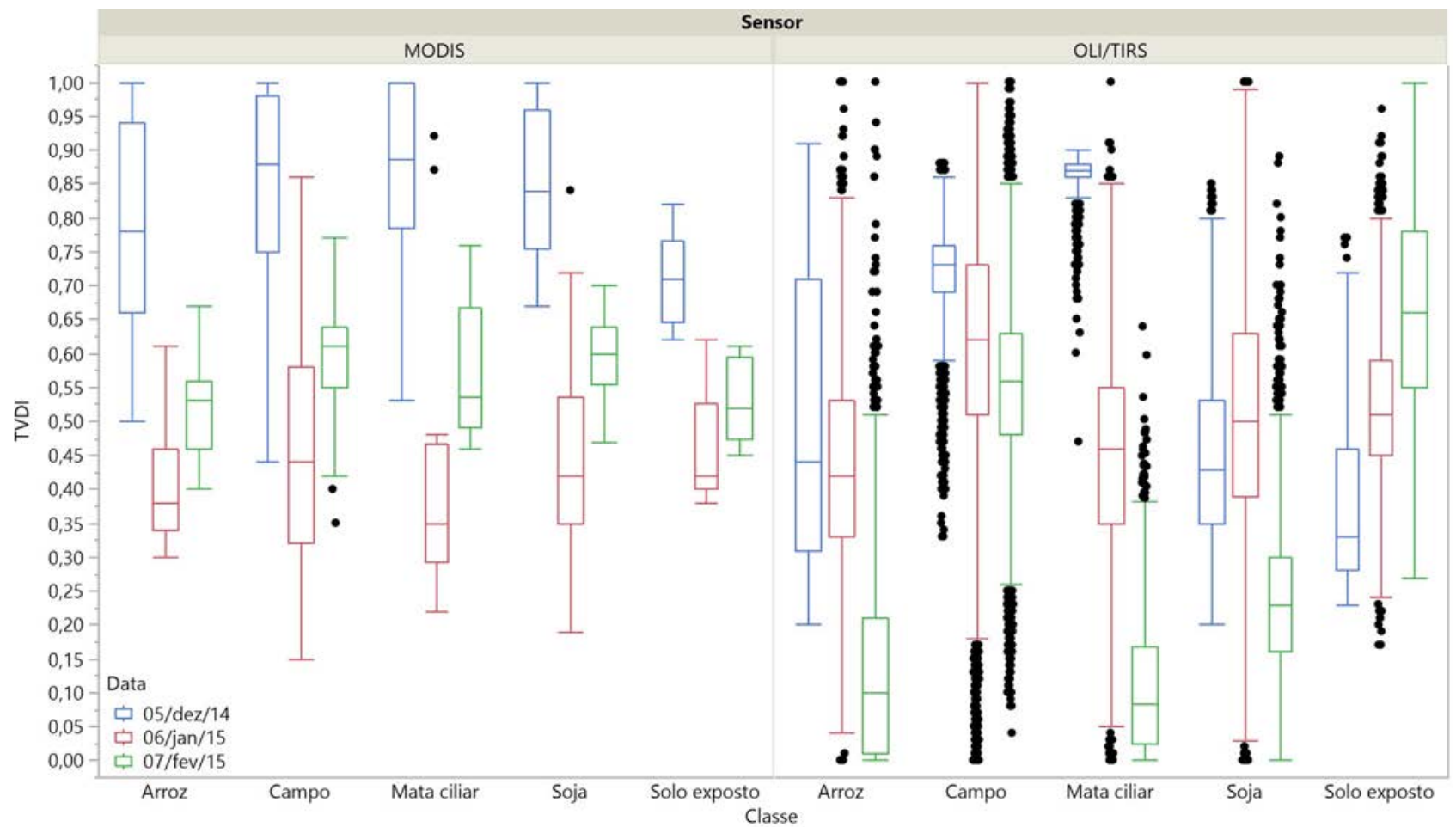

Figura 5 - Distribuição da dispersão dos dados de TVDI das classes de arroz, campo, mata ciliar, soja e solo exposto, para as datas de 5 dez 2014, 6 jan 2015 e 7 fev 2015 para os sensores MODIS e OLI/TIRS na área de estudo.

TVDI ocorreram em fevereiro e não em janeiro conforme se observou nas imagens MODIS (box verdes e vermelhos, respectivamente na Fig. 5). Apenas para solo exposto se verifica os maiores TVDIs em fevereiro.

A análise estatística aplicada aos dados TVDI mostrou que houve efeito altamente significativo da interação tripla, evidenciando padrões de respostas distintas entre sensores, classes e datas. Na comparação entre as médias (Tabela 1), se verificou que para o sensor MODIS não houve efeito da interação classes*datas $(p>0,05)$, mas teve efeito significativo tanto para classes, como também para datas. Como seria esperado para um sensor com resolução espacial moderada $(1 \mathrm{~km})$, existe limites para que com uma única imagem se diferencie classes, porém é possível caracterizar e diferenciar grandes padrões de umidade da superfície, assim como detectar as diferenças que ocorrem ao longo do tempo. Os valores de TVDI de dezembro foram maiores do que o de fevereiro e o de janeiro. As classes campos naturais e soja apresentaram índices superiores ao observado na classe arroz e solo exposto.

Por outro lado, para o sensor OLI/TIRS houve efeito significativo para a interação classes*datas, $(p<0,05)$. OLI/TIRS, com resolução de $30 \mathrm{~m}$, possibilitou monitorar de forma mais detalhada os padrões de cada classe, inclusive diferenciando as mesmas dentro de uma mesma imagem. Analisando cada uma das datas em separado se observou que para 5 dez 2014 todas as classes foram dis-

Tabela 1 - Valores médios de TVDI para os sensores, as classes e as datas das imagens analisadas. Período de dezembro/janeiro/fevereiro na área de estudo.

\begin{tabular}{|c|c|c|c|c|c|c|c|c|}
\hline \multirow[t]{2}{*}{ Classe } & \multicolumn{4}{|c|}{ OLI/TIRS } & \multicolumn{4}{|c|}{ MODIS } \\
\hline & $05 / \mathrm{dez} / 14$ & 06/jan/15 & $07 / \mathrm{fev} / 15$ & Média \pm DP & $05 / \mathrm{dez} / 14$ & 06/jan/15 & $07 /$ fev/15 & Média \pm DP \\
\hline Arroz & $0,50 \mathrm{Ca}$ & $0,43 \mathrm{Db}$ & $0,13 \mathrm{Dc}$ & $0,35 \pm 0,17$ & 0,78 & 0,4 & 0,52 & $0,57 \pm 0,10 \mathrm{~B}$ \\
\hline Campo & $0,72 \mathrm{Ba}$ & $0,61 \mathrm{Ab}$ & $0,55 \mathrm{Bc}$ & $0,63 \pm 0,12$ & 0,84 & 0,47 & 0,59 & $0,64 \pm 0,14 \mathrm{~A}$ \\
\hline Mata ciliar & $0,86 \mathrm{Aa}$ & $0,44 \mathrm{Db}$ & $0,11 \mathrm{Ec}$ & $0,47 \pm 0,12$ & 0,86 & 0,42 & 0,57 & $0,61 \pm 0,15 \mathrm{AB}$ \\
\hline Soja & $0,45 \mathrm{Db}$ & $0,50 \mathrm{Ca}$ & $0,24 \mathrm{Cc}$ & $0,40 \pm 0,14$ & 0,85 & 0,45 & 0,6 & $0,63 \pm 0,11 \mathrm{~A}$ \\
\hline Solo exposto & $0,38 \mathrm{Ec}$ & $0,52 \mathrm{Bb}$ & $0,67 \mathrm{Aa}$ & $0,52 \pm 0,14$ & 0,7 & 0,45 & 0,53 & $0,56 \pm 0,08 \mathrm{~B}$ \\
\hline Média \pm DP & $0,66 \pm 0,09$ & $0,57 \pm 0,17$ & $0,45 \pm 0,12$ & & $0,84 \pm 0,13 a$ & $0,45 \pm 0,17 \mathrm{c}$ & $0,58 \pm 0,07 b$ & \\
\hline
\end{tabular}

Médias seguidas por distintas letras, minúsculas na linha e maiúsculas na coluna, diferem pelo teste Tukey $(\mathrm{p}<0,05)$. 
tintas (Tabela 1). As maiores restrições de umidade estiveram associadas às classes de mata ciliar e campos naturais. Nas matas, talvez alguma oscilação de nível da lâmina de água nos córregos possa explicar esta incoerência, mas isto teria que ser investigado.

Em 6 jan 2015 o sensor não conseguiu diferenciar arroz de mata ciliar, com ambas classes apresentando os menores valores de TVDI, o que é coerente com a presença de água nas lavouras de arroz (sob inundação) e pela proximidade da mata ciliar aos cursos d'água. Já os maiores valores do índice estiveram associados às classes de campos naturais, solo exposto e soja (Tabela 1).

Em 7 fev 2015 novamente todas as classes se diferenciaram (Tabela 1). Os menores valores do índice ocorreram na mata ciliar e arroz e os maiores valores no solo exposto e campos naturais.

\subsection{Parametrização MODIS novembro-maio}

Usando a parametrização envolvendo toda a safra de primavera-verão foi possível caracterizar as variações no TVDI ao longo do tempo. O perfil TVDI-MODIS (Fig. 6) dos alvos analisados (arroz, soja, campo naturais, mata ciliar e solo exposto) apresentou um padrão temporal similar. Os valores foram mais altos em dezembro, quando os déficits hídricos (Fig. 4) coincidiram com a época de implantação das culturas, com os menores NDVI (Fig. 7a), maior presença de solo exposto, e maiores $T_{S}$ (Fig. $7 \mathrm{~b}$ ). Em fevereiro, no período de maior desenvolvimento vegetativo das culturas de primavera-verão, com maior NDVI (Fig. 7a), a elevação do TVDI expressou a ocorrência de déficit hídrico no solo (Fig. 4), ocasionando o fechamento dos estômatos e a consequente elevação da $T_{S}$ (Fig. 7b) (Allen et al. 2006). De março a abril os menores TVDIs foram consequência da finalização do ciclo, com baixos valores de NDVI (Fig. 7a), e das menores $T_{S}$ características do início do outono (Fig. 7b). Observou-se ainda no dia 22 de março a ocorrência de uma queda bastante acentuada no TVDI, o que possivelmente tenha sido decorrência de uma queda da temperatura do ar, em torno de $5^{\circ} \mathrm{C}$, observada na estação meteorológica de Santa Maria.

\section{Conclusões}

$\mathrm{O}$ índice TVDI pode ser obtido usando imagens dos sensores OLI/TIRS e MODIS, os quais apresentam um padrão similar de distribuição no espaço e ao longo do tempo, mas com magnitudes distintas. Na média de todas as datas e classes, o TVDI obtido das imagens MODIS foi superior em 0,128 unidades ao TVDI obtido com o sensor OLI/TIRS, mas com diferenças acentuadas entre estas. As maiores diferenças foram observadas para a classe soja e na primeira data de análise.

Quando utilizado OLI/TIRS é possível um maior detalhamento espacial das condições hídricas das culturas de primavera-verão, dada as diferenças de resolução espacial, na área de estudo nas imagens OLI/TIRS tem-se mais de 270 kpixels, enquanto que nas imagens MODIS, são apenas 265. Já utilizando o TVDI-MODIS é possível monitorar as condições hídricas em escala regional e com maior detalhamento temporal (dada as diferenças de resolução temporal obteve-se 12 imagens compostas no período estudado, enquanto que nas imagens OLI/TIRS foram apenas três imagens disponíveis sem nebulosidade no período).

Os perfis temporais de TVDI-MODIS, são capazes de descrever ao longo do período de análise a condição hídrica ocorrida nos alvos de interesse, portanto estas imagens podem contribuir em um sistema de monitoramento agrometeorológico da área de estudo, especialmente com

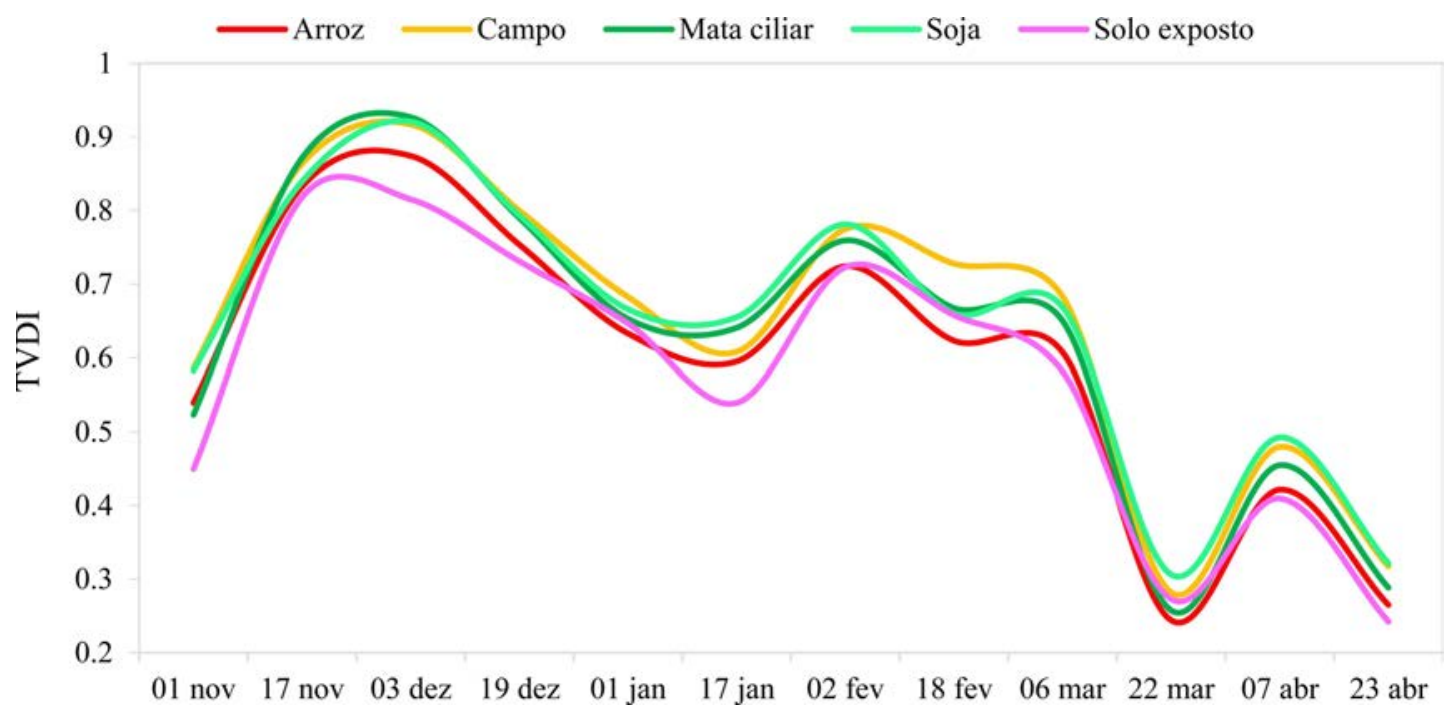

Figura 6 - Perfil médio de TVDI para MODIS nos meses de novembro de 2014 a abril de 2015 para os alvos de arroz, soja, campo, mata ciliar e solo exposto na área de estudo. 

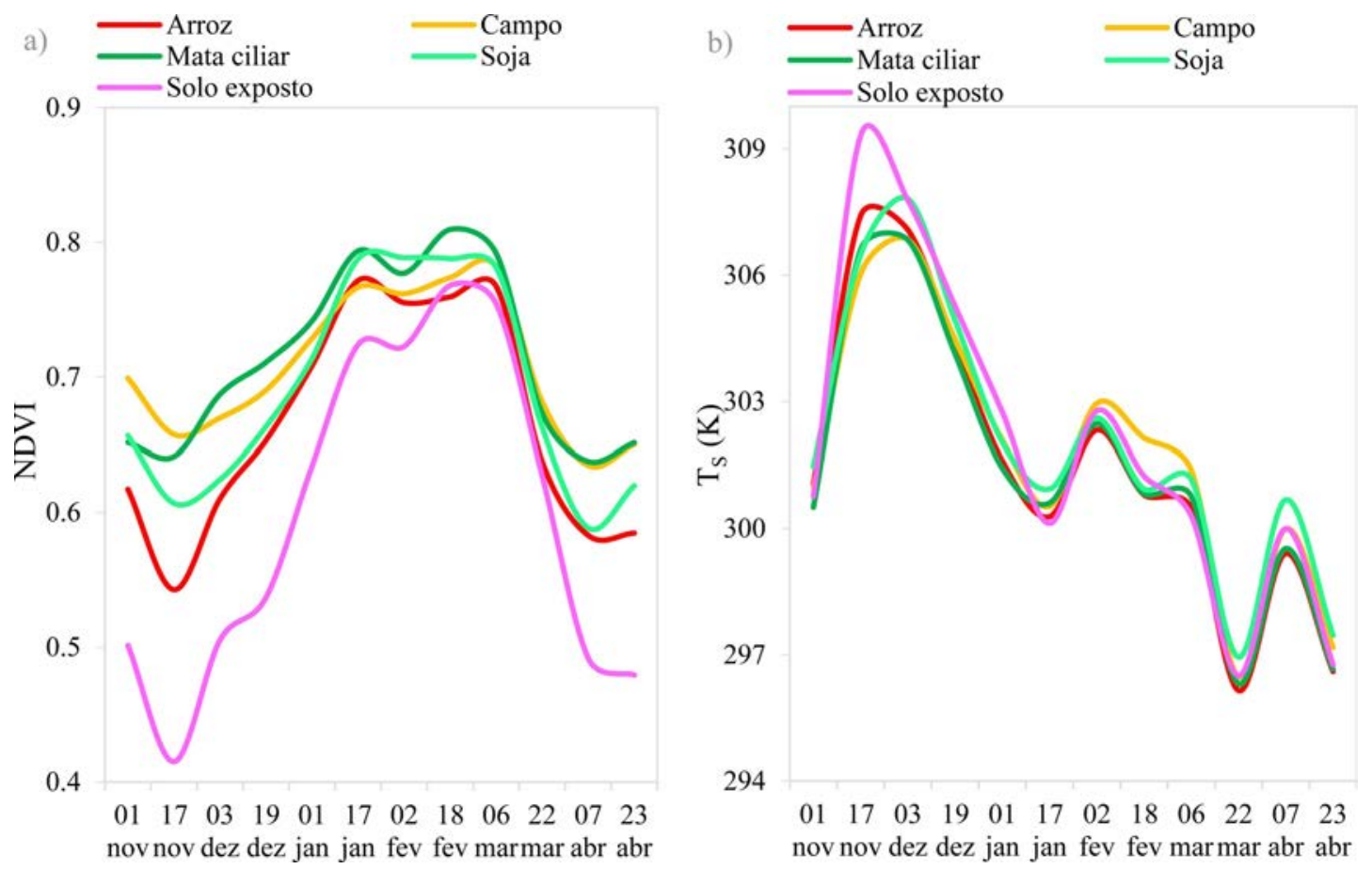

Figura 7 - Perfil médio do NDVI para MODIS (a); perfil médio da temperatura de superfície ( $T_{S}$ ) para MODIS (b). Nos meses de novembro de 2014 a abril de 2015 para os alvos de arroz, soja, campo, mata ciliar e solo exposto na área de estudo.

o atributo temporal, desde que os tamanhos das parcelas sejam compatíveis com a resolução espacial do sensor.

Os resultados na área de estudo evidenciaram que ambos sensores podem, portanto, ser utilizados de forma conjunta, trazendo informações complementares sendo que OLI/TIRS e MODIS apresentam, respectivamente, vantagens em termos de detalhamento espacial e temporal.

\section{Agradecimentos}

O presente trabalho foi realizado com apoio da Coordenação de Aperfeiçoamento de Pessoal de Nível Superior Brasil (CAPES) - Código de Financiamento 001.

\section{Referencias}

ALLEN, R.G.; PEREIRA, L.S.; RAES, D.; SMITH, M. Evapotranspiración del Cultivo: Guías para la Determinación de los Requerimientos de Agua de los Cultivos. In: FAO: Irrigation and Drainage Paper, 56.Roma: FAO. 2006, $323 \mathrm{p}$.

ALVARES, C.A.; STAPE, J.L.; SENTELHAS, P.C.; GONÇALVES, J.L.M.; SPAROVEK, G. Köppen's climate classification map for Brazil. Meteorologische Zeitschrift, v. 22, n. 6, p. 711-728, 2013.

BERLATO, M.A.; CORDEIRO, A.P.A. Sinais de mudanças climáticas globais e regionais, projeções para o século XXI e as tendências observadas no Rio Grande do Sul: uma revisão. Agrometeoros, v. 25, n. 2, p. 273-302, 2017.
CARLSON, T.N.; GILLIES, R.R.; PERRY, E.M. A method to make use of thermal infrared temperature and NDVI measurements to infer surface soil water content and fractional vegetation cover. Remote Sensing Reviews, v. 9, p. 161173, 1994.

CHEN, S.; WEN, Z.; JIANG, H.; ZHAO, Q.; ZHANG, X.; CHEN, Y. Temperature vegetation dryness index estimation of soil moisture under different tree species. Sustainability, v. 7, p. 11401-11417, 2015.

EMATER - Empresa de Assistência Técnica e Extensão Rural. Informativo Conjuntural, 149315 de março de 2017. Disponível em: http://www.emater.tche.br/site/servicos/ informacoes-agropecuarias.php\#conjuntural. Acesso em: 21 mar. 2018.

GUASSELLI, L.A.; SALDANHA, D.L.; SUERTEGARAY, D.M.A.; DUCATI, J.R.; FONTANA, D.C. Macrozoneamento do estado do Rio Grande do Sul. Pesquisas em Geociências, v. 33, n. 1, p. 3-11, 2006.

HOLZMAN, M.E.; RIVAS, R.E.; PICCOLO, M.C. Estimating soil moisture and the relationship with crop yield using surface temperature and vegetation index. International Journal of Applied Earth Observation and Geoinformation, v. 28, p. 181-192, 2014.

JIMÉNEZ-MUÑOZ, J.C.; SOBRINO, J.A.; SKOKOVIC, D.; MATTAR, C.; CRISTÓBAL, J. Land surface temperature retrieval methods from Landsat-8 thermal infrared sensor data. IEEE Geoscience and Remote Sensing Letters, v. 11, p. 1840-1843, 2014.

MALLICK, K.; BHATTACHARYA, B.K.; PATEL, N.K. Estimating volumetric surface moisture content for cropped soils using a soil wetness index based on surface temperature and NDVI. Agricultural and Forest Meteorology, v. 149, p. 1327-1342, 2009. 
NEMANI, R.R.; RUNNING, S.W. Estimation of regional surface resistance to evapotranspiration from NDVI and thermal IR AVHRR data. Journal of Applied Meteorology, v. 28, p. 276-284, 1989.

NICLÒS, R.; CASELLES, V.; COLL. C.; VALOR, E. Determination of sea surface temperature at large observation angles using an angular and emissivity-dependent splitwindow equation. Remote Sensing of Environment, v. 111, p. 107-12, 2007.

ROUSE, J.W.; HAAS, R.H.; SCHELL, J.A.; DEERING, D.W. Monitoring vegetation systems in the Great Plains with ERTS. In: Third Symposium of ERTS, Greenbelt: Maryland, v. 1, p. 309-317, 1973.

SANDHOLT, I.; RASMUSEN, K.; ANDERSEN, J. A simple interpretation of the surface temperature/vegetation index space for assessment of surface moisture status. Remote Sensing of Enviromenmt, v. 79, p. 213-224, 2002.

SAYAGO, S.; OVANDO, G.; BOCCO, M. Landsat images and crop model for evaluating water stress ofrainfed soybean. Remote Sensing of Environment, v. 198, p. 30-39, 2017.

SCHIRMBECK, L.W.; FONTANA, D.C.; SCHIRMBECK, J. Two approaches to calculate the TVDI in the humid subtropical climate of southern Brazil. Scientia Agricola, v. 75 , n. 2, p. $95-172,2018$.
SCHIRMBECK, L.W.; FONTANA, D.C.; SCHIRMBECK, J.; MENGUE, V.P. Uso do índice TVDI e modelo HAND para caracterização de condição hídrica. Irriga, v. 1, p. 76-84, 2017.

THORNTHWAITE, C.W.; MATHER, J.R. The Water Balance. Publications in Climatology. New Jersey: Drexel Institute of Technology, 1955, 104p.

UNIYAL, B.; DIETRICH, J.; VASILAKOS, C.; TZORAKI, O. Evaluation of SWAT simulated soil moisture at catchment scale by field measurements and Landsat derived indices. Agricultural Water Management, v. 193, p. 55-70, 2017.

WANG, C.Y.; QI, S.H.; NIU, Z.; WANG, J.B. Evaluating soil moisture status in China using the temperature-vegetation dryness index (TVDI). Canadian Journal of Remote Sensing, v. 30, p. 671-679, 2004.

ZHANG, F.; ZHANG, L.; SHI, J.; HUANG, J. Soil moisture monitoring based on land surface temperature-vegetation index space derived from MODIS Data. Pedosphere, v. 24, n. 4 , p. $450-460,2014$.

License information: This is an open-access article distributed under the terms of the Creative Commons Attribution License (type CC-BY), which permits unrestricted use, distribution and reproduction in any medium, provided the original article is properly cited. 